\title{
Effect of Acoustic Wave on the Parametric X-ray Generation by Relativistic Electrons in a Crystal
}

\author{
P. N. Zhukova, M. S. Ladnykh, A. G. Mkrtchyan, A. R. Mkrtchyan, and N. N. Nasonov* \\ Belgorod State University, Belgorod, 308007 Russia \\ Institute of Applied Problems in Physics, National Academy of Sciences of Armenia,
}

\begin{abstract}
An exhaustive description of the parametric X-ray (PXR) generation by relativistic electrons in a crystal excited by an acoustic wave is proposed. A principal question as to whether it is possible to enhance the PXR yield under these conditions is considered.
\end{abstract}

Increasing interest in the analysis of high-energy electromagnetic processes in oriented crystals under the action of external factors (e.g., acoustic waves) is related to the possibility of controlling these processes. In particular, the effect of acoustic waves was studied with respect to the coherent bremsstrahlung radiation $[1,2]$, coherent electron-positron pair production in a crystal [3, 4], diffraction of free photons [5, 6], X-ray transition radiation [7], and parametric X-ray (PXR) generation [8-13].

Our previous experimental investigations [12, 13] showed an increase in the PXR yield with the acoustic wave power, but this phenomenon has not been given proper theoretical explanation so far. Polikarpov and Skadorov $[10,11]$ theoretically studied fine dynamic features of the resonant action of acoustic waves on the PXR characteristics, which are manifested only within narrow spectral and angular intervals and, hence, cannot be used to explain the observed PXR yield enhancement $[12,13]$.

In this Letter, we propose a simple kinematic approach that provides an adequate description of the PXR generation by electrons with energies on the order of up to tens megaelectronvolts. The obtained formulas describe PXR emission in a broad range of angles and frequencies and, in particular, provide a simple explanation of the experimentally observed effect $[12,13]$.

Let us consider the PXR generation by a relativistic electron that moves in a crystal with velocity $\mathbf{V}$. The Maxwell equation for the Fourier image of the electromagnetic field excited in the medium is as follows:

$$
\begin{gathered}
\left(k^{2}-\omega^{2}\right) \mathbf{E}_{\omega \mathbf{k}}-\mathbf{k}\left(\mathbf{k} \mathbf{E}_{\omega \mathbf{k}}\right)+\int d^{3} k^{\prime} G\left(\mathbf{k}^{\prime}-\mathbf{k}\right) \mathbf{E}_{\omega \mathbf{k}^{\prime}} \\
=\frac{i \omega e}{2 \pi^{2}} \mathbf{V} \exp \left(-i \mathbf{k}^{\prime} \mathbf{r}_{0}\right) \delta\left(\omega-\mathbf{k}^{\prime} \mathbf{V}\right) \\
G=\frac{Z e^{2}}{2 \pi^{2} m_{1}\left(\mathbf{k}^{\prime}-\mathbf{k}\right)^{2} R^{2}} \sum_{l} \exp \left(i\left(\mathbf{k}^{\prime}-\mathbf{k}\right)\right) \mathbf{r}_{l}
\end{gathered}
$$

where the last term in the left-hand part of the equation describes the induced current of electrons in the medium within the high-frequency approximation ( $\omega \gg I, I$ being the average ionization potential of atoms in the target), which allows the atomic frequencies to be ignored in comparison to $\omega$ in the energycontaining denominators of the formula for atomic polarizability [14] and the asymptotic formula (1) to be used; $Z$ is the number of electrons in the atom, $R$ is the radius of electron screening of the atomic nucleus; and $G$ is a function that describes the individual contributions of all target atoms with coordinates $\mathbf{r}_{7}$.

For the subsequent analysis, it is convenient to represent function $G$ as a sum of the average (refraction) and fluctuation (scattering) components as follows:

$$
G=\bar{G}+\tilde{G}, \quad \bar{G}=\langle G\rangle_{r_{l}}=\omega_{p}^{2} \delta\left(\mathbf{k}^{\prime}-\mathbf{k}\right),
$$

where $\omega_{p}$ is the plasma frequency. Substituting expression (2) into Eq. (1) and solving the obtained equation by iterations with respect to powers of $\tilde{G}$ (which yields a well-known formula for the Coulomb field of a fast electron in a medium for the zero-order approximation and an expression for the radiation field in the next approximation), we arrive at the following 


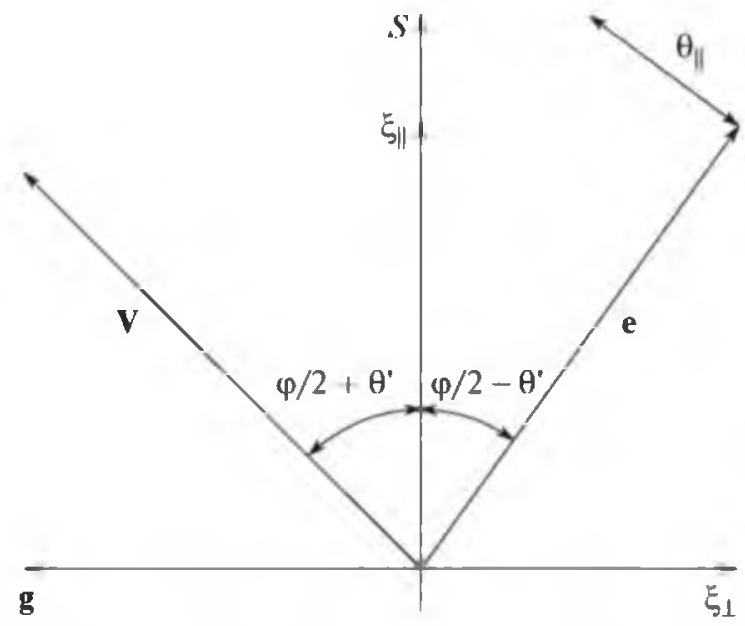

lïg. 1. Geometry of PXR emission: (S) rellecting crystallographic plane set by the reciprocal lattice vector $g ;(\varphi)$ preset PXR emission angle; $\left(\theta^{\prime}\right)$ orientation angle determined using a goniometer; $\left(\theta_{\|}\right)$component of the twodimensional observation angle $\mathbf{\theta}\left(\mathbf{n}=\mathbf{e}\left(1-\theta^{2} / 2\right)+\mathbf{\theta}\right.$, el $=0$ ), which is lying in the scattering plane; $\left(\xi_{\|,}, \xi_{\perp}\right)$ components of the wave vector of the acoustic wave.

expression for the spectral and angular distribution of the emitted radiation photons:

$$
\begin{aligned}
& \omega \frac{d N}{d \omega d \Omega}=e^{2} \omega^{2} \int \frac{d^{3} k_{1}}{k_{1}^{2}+2 \omega \sqrt{\varepsilon} \mathbf{n} \mathbf{k}_{1}} \frac{d^{3} k_{2}}{k_{2}^{2}+2 \omega \sqrt{\varepsilon} \mathbf{n k}} \\
& \times\left\langle\bar{G}\left(\mathbf{k}_{1}\right) \tilde{G} \tilde{i}^{*}\left(\mathbf{k}_{2}\right)\right\rangle\left\langle\exp \left(i\left(\mathbf{k}_{1}-\mathbf{k}_{2}\right) \mathbf{r}_{1}\right)\right\rangle \\
& \times\left(V-\frac{k_{1}}{\omega \varepsilon}-n\left(n V-\frac{n k_{1}}{\omega \varepsilon}\right)\right)\left(V-\frac{k_{2}}{\omega \varepsilon}-n\left(n V-\frac{n k_{2}}{\omega \varepsilon}\right)\right) \\
& \times \delta\left(\omega(1-\sqrt{\varepsilon} \mathbf{n V})-\mathbf{k}_{1} \mathbf{V}\right) \delta\left(\omega(1-\sqrt{\varepsilon} \mathbf{n V})-\mathbf{k}_{2} \mathbf{V}\right) .
\end{aligned}
$$

Here, the angle brackets imply averaging over the positions of atoms in the target and the radius $\mathbf{r}_{0}$ that determines the electron trajectory within the crystal lattice cell; $\mathbf{n}$ is the unit vector in the direction of radiation propagation; $\varepsilon(\omega)=1-\omega_{p}^{2} / \omega^{2}$ is the ordinary dielectric permittivity in the $\mathrm{X}$-ray spectral range, which is given by Eqs. ( 1 ) and (2) and condition $\omega \gg I$.

The averaging is performed using the following formula:

$$
\mathbf{r}_{l}=\mathbf{R}_{l}+\mathbf{u}_{l}+\mathbf{a} \sin \left(\xi \mathbf{R}_{l}\right),
$$

where $\mathbf{R}_{\text {i }}$ is the equilibrium coordinate of an atom (evidently, this sequence is periodic), $\mathbf{u}_{\text {l }}$ is the thermal atomic displacement, and $\mathbf{a}$ and $\xi$ are the acoustic wave amplitude and wave vector, respectively. The result of averaging is conventionally presented as a sum of the incoherent and coherent components. The coherent component that is of interest in this consideration represents a sun of independent terms corresponding to various vectors of the reciprocal lattice of the crystalline target. The term that describes the coherent diffraction of the Coulomb field of an elec- tron on a perturbed crystallographic plane determined by vector $\mathrm{g}$ is as follows:

$$
\begin{gathered}
\omega \frac{d N_{\varepsilon}}{d i d \omega d \Omega} \approx \frac{e^{2} \omega^{2} \omega_{p}^{4}}{2 \pi} \sum_{p} J_{p}^{2}\left(\mathbf{g}_{p} \mathbf{a}\right) \\
\times \frac{\left(\mathbf{V}-\mathbf{g}_{p} / \omega \varepsilon\right)^{2}-\left(\mathbf{n V}-\mathbf{n} \mathbf{g}_{p} / \omega \varepsilon\right)^{2}}{\left(\mathbf{g}_{p}^{2}+2 \omega \sqrt{\varepsilon} \mathbf{n} \mathbf{g}_{p}\right)^{2}} \\
\times \delta\left(\omega(1-\sqrt{\varepsilon} \mathbf{n V})-\mathbf{g}_{p} \mathbf{V}\right),
\end{gathered}
$$

where $\mathbf{g}_{p}=\mathbf{g}-p \xi$ and $\omega_{g}^{2}=\omega_{p}^{2} \exp \left(-g^{2} u^{2} / 2\right)(1+$ $\left.g^{2} R^{2}\right)^{-1}$.

For the subsequent analysis, we introduce the variables $0_{11}$ and $0_{1}$, which describe the angular distribution of X-ray photons, and the angle $\theta^{\prime}$ that describes the angular dependence of the PXR yield. The geometry of PXR emission is depicted in Fig. 1. In the new variables, formula (5) eventually appears as follows:

$$
\begin{gathered}
\omega \frac{d N_{g}}{d l d \omega d \Omega} \approx \frac{e^{2} \omega_{g}^{4}}{\pi g^{2}} \sum_{p} J_{p}^{2}(\mathbf{g a}) \\
\times \frac{0_{\perp}^{2}+\left(\theta_{\|}+2 \theta^{\prime}-2 p \frac{\xi_{\|}}{g}\right)^{2} \cos ^{2} \varphi}{\left(\rho^{2}+\theta_{\perp}^{2}+\left(\theta_{\|}+2 \theta^{\prime}-2 p \frac{\xi_{\|}}{g}\right)^{2}\right)^{2}} \\
\times \delta\left(\omega-\omega_{\mathrm{B}}\left(1+\left(0_{\|}+0^{\prime}-\frac{p \xi_{V}}{g \cos \frac{\varphi}{2}}\right) \cot \frac{\varphi}{2}\right)\right),
\end{gathered}
$$

where $\xi_{V}=\xi_{\|} \cos \frac{\varphi}{2}-\xi_{\perp} \sin \frac{\varphi}{2}$ is the component of the wave vector of the acoustic wave, which is parallel to the electron velocity, $\omega_{\beta}$ is the Bragg frequency. $\rho^{2}=$ $\gamma^{-2}+\omega_{p}^{2} / \omega^{2}$. and $\gamma$ is the Lorentz factor of electron. Note that the simple kinematic formula (6) is valid in a broad range of electron energies $m \gamma<m \gamma_{*}=$ $m \omega_{\mathrm{B}} / \omega_{p} \leq 100 \mathrm{MeV}|15|$.

Now let us consider the obtained expression and use it for a qualitative interpretation of the experimental results $|12,13|$ (unfortunately, the available data are insufficient for a quantitative description). An analysis of Eq. (6) shows that the aforementioned PXR yield enhancement is related to the bending oscillations of the reflecting plane (only the component of the acoustic wave amplitude that is perpendicular to this plane enters into Eq. (6)). Naturally, it is the component $\xi$ parallel to the reflecting plane that leads to the angular modulation of emitted radiation, since the $\xi_{1}$ component does not lead to bending of the reflecting plane.

According to Eq. (6), the periodic bending of the system of parallel atomic planes determined by vector 


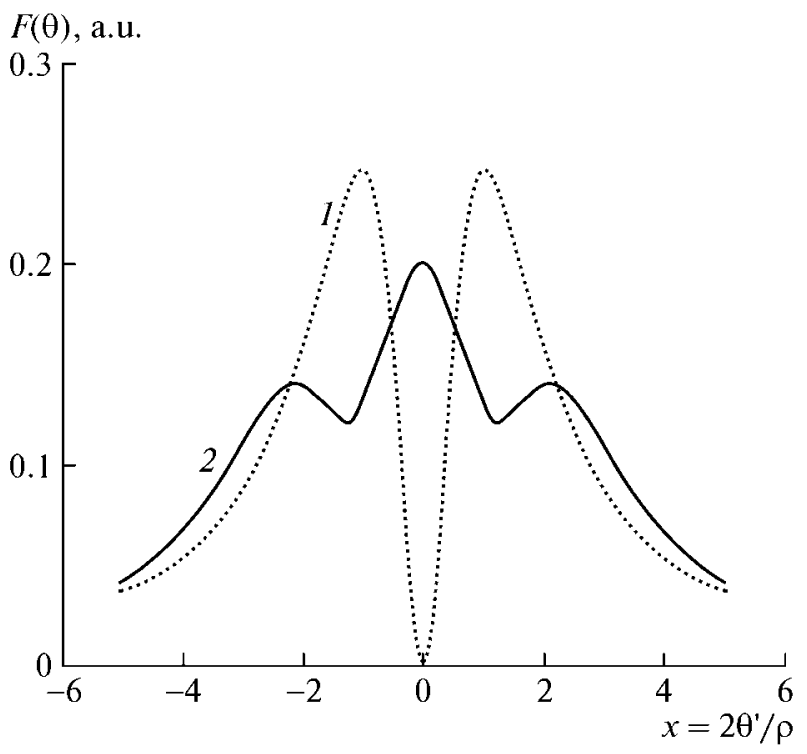

Fig. 2. Orientation dependence of the angular distribution of the PXR photon density ( $I$ ) for unperturbed crystal ( $y=$ $\left.\mathbf{g a}=2.9, z=2 \xi_{\|} / g \rho=0\right)$ and $(2)$ in the presence of acoustic wave $\left(y=\mathbf{g a}=2.9, z=2 \xi_{\|} / g \rho=0.6\right)$.

g can be considered as replacement of one atomic plane by a series of lower-density planes rotated relative to each other. Then, the final angular distribution of the emitted PXR photons consists of a set of reflections with the same structure and various amplitudes, which are shifted relative to each other. Apparently, the angular density of radiation can be significantly changed under the action of the acoustic wave, but the total PXR yield integrated over angles and frequencies coincides with the yield from the unperturbed crystal (this statement follows from the condition $\sum_{p} J_{p}^{2}($ ga $)=1$ ).

In order to illustrate the laws under consideration, let us analyze the orientation dependence of a strictly collimated PXR beam $\left(\theta_{\|}, \theta_{\perp}<\rho\right)$. In this case, Eq. (6) yields

$$
\frac{d N_{g}}{d t d \Omega} \approx F\left(\theta^{\prime}\right) \sim \sum_{p} J_{p}^{2}(y) \frac{(x-p z)^{2}}{\left(1+(x-p z)^{2}\right)^{2}},
$$

where $x=2 \theta^{\prime} / g \rho, y=\mathbf{g a}$, and $z=2 \xi_{/} / g \rho$. Figure 2 shows plots of the function $F$ calculated using formula (7) for different values of parameters. These curves demonstrate the possibility of a sharp change in the angular distribution of PXR photons under the action of a control acoustic wave. In particular, it is possible to provide for a significant increase in the radiation yield as measured by a detector with a small angular aperture $\left(\theta_{D}<\rho\right)$, which is oriented in the direction of Bragg scattering. This possibility explains the results obtained in [12, 13].

It should be noted that function $F$ in Eq. (7) and the curves in Fig. 2 are universal (the crystal is only char-

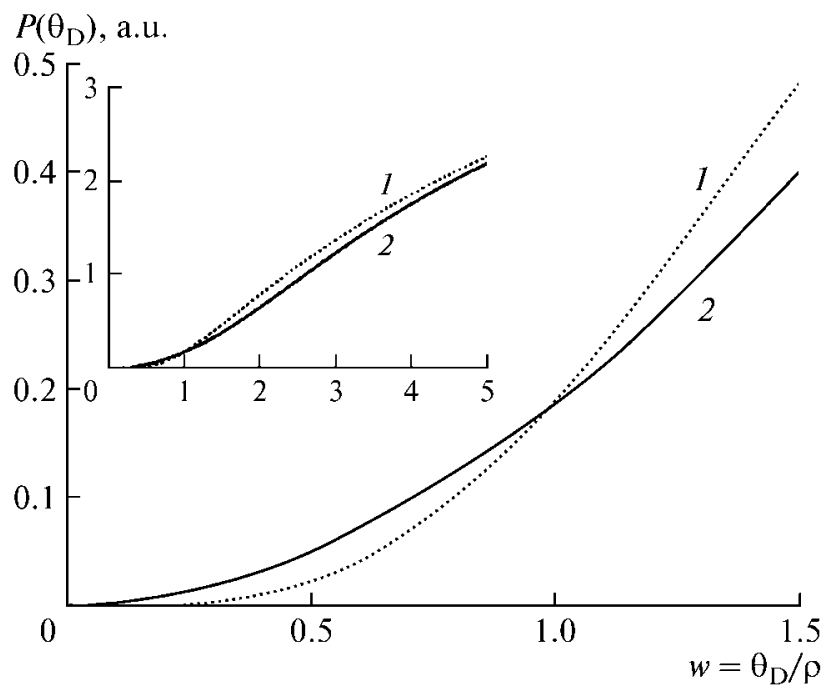

Fig. 3. Dependence of the PXR yield on the collimator size $w$ for ( 1 ) unperturbed crystal $\left(y=\mathbf{g a}=0, z=2 \xi_{\|} / g \rho=\right.$ $0)$ and (2) in the presence of acoustic wave $(y=$ ga $=2, z=$ $\left.2 \xi_{\|} / g \rho=0.5\right)$. The inset shows behavior of the PXR yield on a greater scale.

acterized by the reciprocal lattice vector $\mathbf{g}$, while particular cases are specified for calculations of the amplitude a and wave vector $\xi$ of the acoustic wave). Note also that the parameters $y=$ ga and $z=2 \xi_{\mid} / g \rho$ of these curves are quite accessible. For example, with the lattice parameter $l=5 \times 10^{-8} \mathrm{~cm}$, acoustic wavelength $\lambda=5 \times 10^{-5} \mathrm{~cm}$, and electron energy $100 \mathrm{MeV}$, the wave amplitude must be $a \approx 0.5 l$ to provide for the plane bending by an angle as small as $0.5 \mathrm{mrad}$.

Another interesting issue is the dependence of the PXR intensity on the collimator size $\theta_{D}$. Assuming for the simplicity that $\varphi \ll 1$ in Eq. (6) and integrating this equation with respect to the frequencies and angles of observation, we eventually obtain the following formula:

$$
\begin{gathered}
\frac{d N_{g}}{d t} \approx P\left(\theta_{\mathrm{D}}\right) \sim \sum_{p} J_{p}^{2}(y)\left[\ln \left(\frac{1}{2}(A(w)+B(w))\right)\right. \\
\left.-\frac{1}{2}\left(\frac{A(w)}{B(w)}-\frac{A(0)}{B(0)}\right)+\frac{1}{B(w)}-\frac{1}{B(0)}\right], \\
A(w)=1+w^{2}-4 p^{2} z^{2} \\
B(w)=\sqrt{\left(1+w^{2}-4 p^{2} z^{2}\right)^{2}+16 p^{2} z^{2}},
\end{gathered}
$$

where $w=\theta_{\mathrm{D}} / \rho$. The curves plotted in Fig. 3 show that an increase in the PXR yield is only possible provided that the collimator size does not exceed the width of the characteristic dip in the angular distribution of the PXR photon density for an unperturbed crystal. Therefore, the presence of this dip is a necessary condition for manifestation of the PXR yield enhancement under the action of acoustic waves $[12,13]$. 
The inset to Fig. 3 presents the $P(w)$ curves constructed in a broader range of variation of the argument, which show that (in agreement with the above assumptions), the PXR yield into a sufficiently wide collimator is independent of the acoustic wave parameters.

Thus, the above analysis provides an exhaustive description of the effect of acoustic waves on the PXR generation by relativistic electrons in a crystal, which is based on simple kinematic formula (6). It is demonstrated that the total PXR yield is independent of the wave parameters, but the yield into a limited collimator can be significantly changed under the acoustic wave action, which explains the experimentally observed effect [12, 13].

Acknowledgments. The authors are grateful to the referee for constructive remarks.

This study was supported by the Russian Foundation for Basic Research (project no. 09-02-97528) and the Ministry of Education and Science of the Russian Federation (project nos. GK 02.740.11.0545 and GK P2317).

\section{REFERENCES}

1. A. A. Saharian, A. R. Mkrtchyan, V. V. Parazian, and L. Sh. Grigoryan, Mod. Phys. Lett. A 19, 99 (2004).

2. N. F. Shulga, V. V. Boiko, Pis'ma Zh. Éksp. Teor. Fiz. 84, 366 (2006) [JETP Lett. 84, 305 (2006)].
3. A. R. Mkrtchyan, A. A. Saharian, L. Sh. Grigoryan, and B. V. Khachatryan, Mod. Phys. Lett. A 18, 2581 (2002).

4. V. V. Parazian, J. Phys. Cond. Matter 21, 185401 (2009).

5. I. R. Entin, Zh. Éksp. Teor. Fiz. 77, 214 (1979) [Sov. Phys. JETP 50, 110 (1979)].

6. A. V. Kolpakov and Yu. P. Khapachev, Kristallografiya 18, 474 (1973) [Sov. Phys. Crystallogr. 18, No. 3 (1979)].

7. L. Sh. Grigoryan, A. H. Mkrtchyan, and A. A. Saharian, Nucl. Instr. Meth. Phys. Res. B 145, 197 (1998).

8. V. G. Baryshevskii and I. V. Polikarpov, Zh. Éksp. Teor. Fiz. 94, 109 (1988) [Sov. Phys. JETP 67 (1988)].

9. A. R. Mkrtchyan, M. A. Aslanyan, A. H. Mkrtchyan, and L. Sh. Grigoryan, Phys. Lett. A 152, 293 (1991).

10. I. V. Polikarpov and V. V. Skadorov, Phys. Status Solidi (b) 143, 11 (1987).

11. I. V. Polikarpov and V. V. Skadorov, Izv. Akad. Nauk BelSSR, Ser. Fiz.-Mat. Nauk, No. 3, 83 (1988).

12. A. R. Mkrtchyan, H. A. Aslanyan, A. H. Mkrtchyan, et al., Solid State Commun. 74, 187 (1991).

13. A. R. Mkrtchyan, A. G. Mkrtchyan, A. A. Aslanyan, et al., Izv. Nats. Akad. Nauk Arm., Fiz. 47, 282 (2005).

14. V. M. Agranovich and V. L. Ginzburg, Crystal Optics with Allowance for Spatial Dispersion (Nauka, Moscow, 1979) [in Russian].

15. N. Nasonov and P. Zhukova, Rad. Phys. Chem. 75, 1409 (2006). 